\title{
EXPERIMENTS WITH LIQUID BRIDGES IN SIMULATED MICROGRAVITY
}

\author{
J. MESEGUER, L.A. MAYO, J.C. LLORENTE and A. FERNANDEZ \\ Laboratorio de Aerodinámica, ETSI Aeronáuticos, Universidad Politécnica, 28040 Madrid, Spain
}

Received 10 April 1985; manuscript received in final form 30 September 1985

\begin{abstract}
A feature of stability diagrams of liquid bridges between unequal disks subjected to small axial gravity forces is that, for each separation of disks, there is a value of microgravity for which an absolute minimum volume limit is reached. The dependence of such microgravity values on the liquid bridge geometry has been experimentally checked by using the neutral buoyancy technique, experimental results being in complete agreement with theoretical ones. Analytical background assuring the experimental procedure used is presented, and a second order analytical expression for the equilibrium interface shapes is also calculated.
\end{abstract}

\section{Introduction}

A liquid bridge consists of a volume of liquid held by surface tension forces between two solid disks (fig. 1). Equilibrium shapes and stability limits of liquid bridges have drawn the attention of numerous scientists during the last two decades, specially about the stability limits of minimum volume (a short review of the state of the art in this field can be found in refs. [1-3]). It is well known that for each separation of disks there is a minimum volume of liquid for which a stable liquid bridge can be formed. The dependence of such minimum volume stability limits on non-symmetric effects like an axial microgravity and unequal disks has been studied in refs. [4-6] for the case of long liquid bridges. Available results show that each one of these effects separately decrease the stability of the liquid column (the volume must be increased or the disk separation decreased to keep a stable configuration) but both effects combined can cancel or, in other words, each of these effects can be a stabilizer for the remaining one. This behavior is summarized in fig. 2, where a typical stability diagram for a liquid bridge between unequal disks is shown. According to this plot, for each value of the slenderness $\Lambda=$

* In the following, unless otherwise stated, all lengths are made dimensionless with $R_{0}$ (see fig. 1), volumes with $R_{0}^{3}$ and

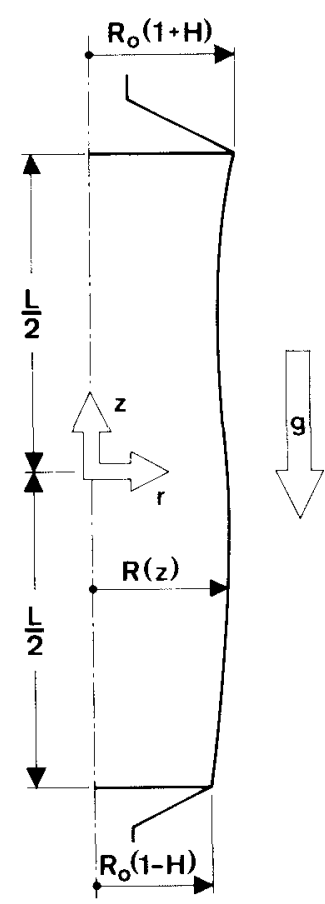

Fig. 1. Geometry and coordinate system for the liquid bridge problem.

pressures with $\sigma / R_{0}, \sigma$ being the surface tension. Bond number is defined as $B=\rho g R_{0}^{2} / \sigma$, where $\rho$ stands for the liquid density and $g$ for the acceleration due to axial microgravity. 


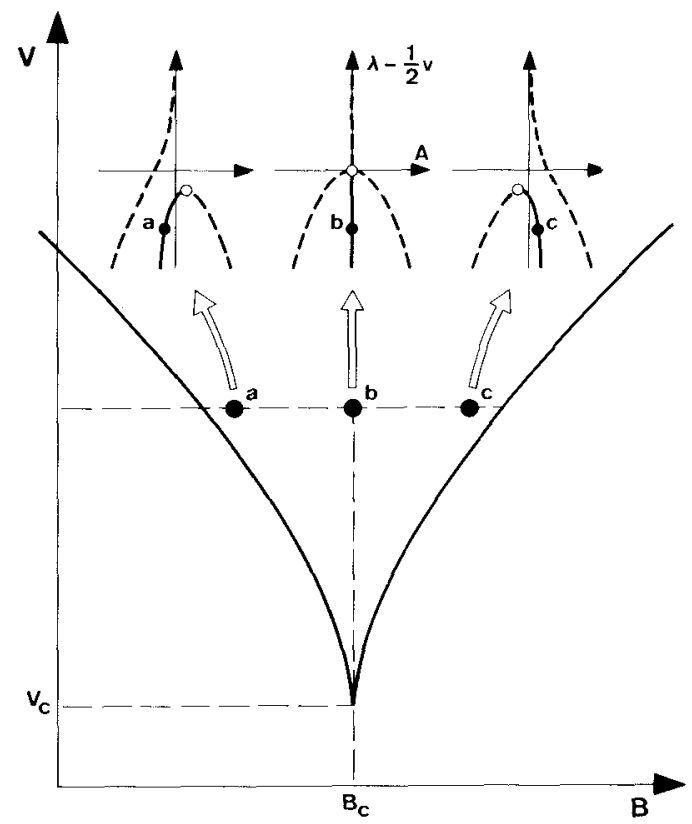

Fig. 2. Stability diagram of liquid bridges between unequal disks. For each value of the slenderness $\Lambda$ and the disk radius ratio $K$ the minimum stable volume $V$ depends on the Bond number $B$ as sketched in the figure. For each couple $\Lambda, K$ there is an absolute minimum volume stability limit $V_{\mathrm{c}}$ which is reached at $B=B_{\mathrm{c}}$. The inserts placed at the top show the bifurcation diagrams ( $A$ versus $\lambda-{ }_{2}^{1} v$, see eqs. (9)) of stable liquid bridge configurations represented by the points labeled $a, b$ c. In these inserts, solid or dashed lines indicate stable or unstable equilibrium shapes, respectively.

$L /\left(2 R_{0}\right) *$ and the disk radius ratio $K=(1-$ $H) /(1+H)$ there is a value of the Bond number $B_{\mathrm{c}}$ for which an absolute minimum-volume stability limit $V_{c}$ is reached.

Numerical results presented in ref. [6] show the dependence of the liquid-bridge interface shapes at stability limits on the Bond number. When $B>B_{\mathrm{c}}$, the neck of the interface is closer to the top disk (the larger disk). As $B$ decreases, it moves towards the bottom disk, the neck being approximately at the middle plane parallel to the disks just when $B=B_{\mathrm{c}}$, at least for the values of $\Lambda$ and $K$ analyzed in ref. [6]. Since a similar behavior can be expected in the case of liquid bridge configurations far from stability limits, one can assume that a stable liquid column will have the neck at the middle plane when $B=B_{\mathrm{c}}$, which provides a criterion to evaluate, either analytical or experimentally, the dependence of $B_{\mathrm{c}}$ on $A$ and $K$. In fact, the follow- ing expression based on the middle-plane hypothesis

$B_{\mathrm{c}}=H /(\Lambda-\sin \Lambda)$,

where $H=(1-K) /(1+K)$, was calculated in ref. [7] from a linear expression for the equilibrium interface shapes. Analytical results obtained from eq. (1) agree with available numerical ones within the range of validity of a linear analysis, which validates the assumption stated on the middle plane.

This paper is devoted to the experimental determination of the dependence of $B_{\mathrm{c}}$ on $\Lambda$ and $K$. Previously an analytical demonstration of the validity of the middle-plane hypothesis for long liquid bridges is presented.

Experiments have been performed by simulating microgravity by using the neutral buoyancy technique, with one liquid surrounded by a second with which it is immiscible but of almost the same density [2,8-14]. In Plateau tank experiments, the Bond number is defined as $B=\Delta \rho g R_{0}^{2} / \sigma$, where $\Delta \rho$ stands for the difference between working and surrounding liquid densities. We used a dimethyl silicone oil as working liquid and a mixture of methanol and distilled water as surrounding liquid. Since both liquids have different thermal expansion coefficients, the variation in liquid temperatures causes variation of the Bond number.

In each of the experiments the following procedure was performed: once a liquid bridge of the appropriated slenderness and volume is formed, the tank is slowly heated (or cooled). As the Bond number changes, the liquid bridge interface varies and the neck moves along the liquid column. From time to time photographs of the interface are taken. The neck position is directly measured from photographs whereas the Bond number is calculated by fitting the experimental interface shapes to an analytical expression. Then, the neck position is plotted versus the Bond number, and the value of $B_{\mathrm{c}}$ is obtained.

Two values of the disk radius ratio ( $K=0.897$ and $K=0.8$ ) and, for each one of these values of $K$, liquid bridges with four different slendernesses (ranging from 2.8 to 1.8 ) have been considered. For redundancy, each liquid bridge configuration has been tested at least twice, changing the volume of liquid from one to another experiment. 


\section{Analytical background}

The results presented in this section are derived from previous analytical results already published in refs. $[5,15]$, where the bifurcation from stable to unstable equilibrium interface shapes of long liquid bridges is studied through a perturbation analysis. The differential equation governing the interface of an axisymmetric liquid bridge between unequal disks subjected to a small axial gravity force is

$$
-\frac{1}{2}\left(2 S+S_{z}^{2}-S S_{z z}\right)\left(S+\frac{1}{4} S_{z}^{2}\right)^{-3 / 2}-B z+P=0,
$$

where $S(z)=R^{2}(z)$ is proportional to the crosssectional area of each slice of the liquid bridge and $P$ is a constant related to the origin of pressures.

Boundary conditions state the value of $S$ at the disks

$S( \pm \Lambda)=(1 \pm H)^{2}$

and the volume of liquid

$V=\pi \int_{-\Lambda}^{\Lambda} S \mathrm{~d} z$

Since we are interested in the stability of liquid bridges with slenderness close to $\Lambda=\pi$ and volumes close to the "cylindrical volume" ( $V=$ $2 \pi \Lambda$ ), the following asymptotic expansions are introduced $[5,15]$

$\Lambda=\pi(1+\epsilon \lambda)$,

$z=x(1+\epsilon \lambda)$,

$S=1+\epsilon^{1 / 2} s_{1}+\epsilon S_{2}+\epsilon^{3 / 2} s_{3}+\ldots$,

$P=1+\epsilon^{1 / 2} p_{1}+\epsilon p_{2}+\epsilon^{3 / 2} p_{3}+\ldots$,

$B=\epsilon^{3 / 2} b$,

$H=\epsilon^{3 / 2} h$,

$V=2 \pi \Lambda(1+\epsilon v)$.

After introducing expressions (5) in eqs. (2), (3) and (4), and equating equal power terms of $\epsilon$, a sequence of problems results.

The solution of the $\epsilon^{1 / 2}$ order problem gives

$s_{1}=-A \sin x$,

where $A$ is an unknown constant measuring the amplitude of the non-symmetric modes, either stables or unstables.

The $\epsilon$ order problem yields

$s_{2}=v(1+\cos x)$,

which is a symmetric, volume-dependent term.

The value of the unknown amplitude $A$ is determined in the $\epsilon^{3 / 2}$ order problem, where the following expression for $s_{3}$ is obtained

$$
\begin{aligned}
s_{3}= & -\left[\left(\lambda-\frac{1}{2} v\right) A+\frac{3}{16} A^{3}\right] x \cos x-\frac{1}{64} A^{3} \sin 3 x \\
& +2 b x+C \sin x+D \cos x .
\end{aligned}
$$

The fulfillment of boundary conditions (3) yields $D=0$ and

$\left(\lambda-\frac{1}{2} v\right) A+\frac{3}{16} A^{3}+2 b=2 h / \pi$,

which determines the amplitude of the interface deformation $A$. The number of real roots of $(9)$ depends on the sign of the discriminant, as it is well known from cubic equation solution, the bifurcation point appearing when

$\left[\frac{16}{3}(b-h / \pi)\right]^{2}=\left[\frac{16}{9}\left(\frac{1}{2} v-\lambda\right)\right]^{3}$.

Furthermore, the nature of the bifurcation represented by eq. (9) depends on the sign of the term $\epsilon^{3 / 2}(b-h / \pi)=B-B_{c}$, as sketched in fig. $2 *$. Bellow the stability limits the amplitude of the stable non-symmetric modes becomes $A=0$ when $B=B_{\mathrm{c}}$, whereas $B \neq B_{\mathrm{c}}$ means $A \neq 0$. Therefore, when $B=B_{\mathrm{c}}$, the stable equilibrium interface shapes are determined, up to the $\epsilon$ order approximation, by the volume-dependent term only (eq. (7)). Since the minimum (or maximum) on a symmetric function is in the middle point, long liquid bridges will have the neck at the middle plane parallel to the disks just when $B=B_{\mathrm{c}}$.

\section{Apparatus}

Experiments have been performed in an apparatus designed as Plateau Tank Facility (PTF) already described in refs. $[2,16]$. A dimethyl sili* This analysis gives $B_{\mathrm{c}}=H / \pi$; the same value results from
eq. (1) when $A=\pi$. 
cone oil (Rhodorsil 47 V20) with viscosity 20 times that of water and density $\rho=954 \pm 0.5 \mathrm{~kg} \mathrm{~m}^{-3}$ has been used as working liquid, and a mixture of methanol and distilled water as the surrounding liquid. To improve interface visibility the dimethyl silicone oil was slightly dyed with yellow aniline.

The tank, as sketched in fig. 3 , is $140 \mathrm{~mm} \times 140$ $\mathrm{mm} \times 60 \mathrm{~mm}$, with the sides made of $2 \mathrm{~mm}$ thick glass and the bottom of $10 \mathrm{~mm}$ thick Perspex. Both disks are made of Perspex, in the shape of a frustum cone, to provide sharp edges. The injection and removal of working fluid is done through a $4 \mathrm{~mm}$ diameter hole in the centre of the upper disk. The working surface of the bottom disk is flat, whereas the one of the feeding disk presents a slight conicity, the purpose of which is to facilitate the evacuation through the injection hole of the air bubbles trapped into the liquid bridge. Working fluid injection and removal is done with a calibrated syringe, the piston of which is driven by a variable speed electric motor. Liquid displaced by the piston passes through the filling duct. In between, a three-way valve with a purge duct is connected. The purge duct is placed aiming to trap air bubbles coming from the upper disk.

A magnetic stirrer at one side of the tank helps to keep uniform temperature and alcohol composition. Background illumination consists of a $60 \mathrm{~W}$ blue glass lamp. Very close to the rear face of the tank a translucent grid provides diffuse illumination and a reference frame for interface shape measurements. A photo camera, placed $70 \mathrm{~cm}$ away, is used for image recording.

Background illumination is used to heat the tank, the rate of heating being controlled by placing appropriate water filters between the blue lamp and the translucent grid. Cooling is achieved by setting off the background illumination and by refrigerating the tank with a cool air stream. The temperature of the surrounding liquid is continuously measured by using a $\pm 0.1^{\circ} \mathrm{C}$ precision thermometer.

One of the problems arising when a methanol-water mixture is used as surrounding liquid is the existence of a density gradient along the tank. This phenomenon was experimentally
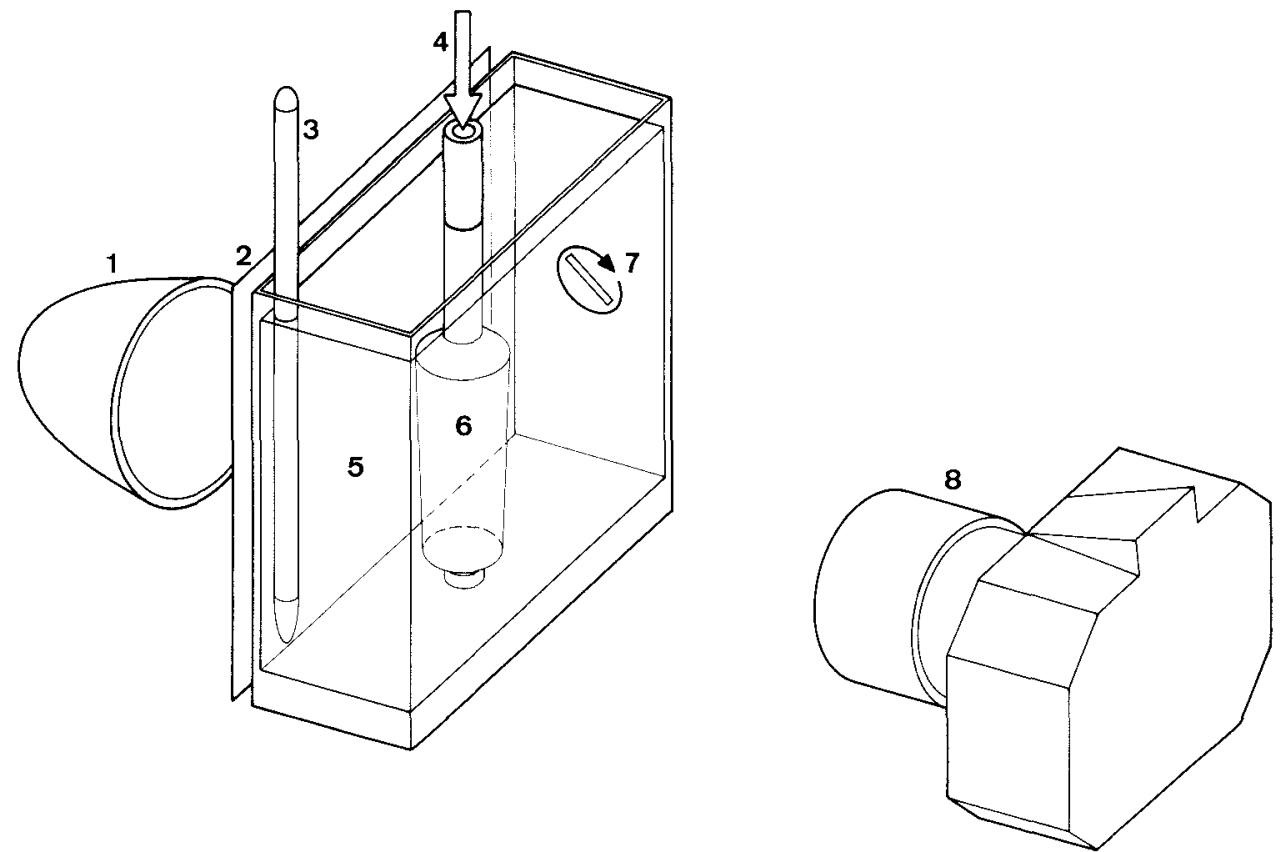

Fig. 3. Sketch of the experimental apparatus: (1) light source, (2) translucent grid, (3) thermometer, (4) filling duct, (5) Plateau tank, (6) liquid bridge, (7) magnetic stirrer, (8) camera. 
studied by Tagg et al. [17] and a density gradient as high as $50 \mathrm{~kg} \mathrm{~m}^{-4}$ was found with their experimental configuration, although these authors did not state whether the density gradient was due to inhomogeneities in the mixture, or to the significant vertical temperature gradient existing in their experiment.

Since we were aware of this problem, during our experiments the magnetic stirrer was intermittently running to avoid density gradients in the surrounding liquid. Such stirrer causes a centrifugal flow of the surrounding liquid (as it can be easily observed by using small working liquid drops as tracers) which prevents density or temperature gradients. Certainly, if the stirrer speed is high enough, the surrounding liquid flow may distort the liquid bridge interface; however, it has been experimentally checked that there is a range of low stirrer speeds in which an effective surrounding liquid mixing is achieved whereas surrounding liquid flow does not appreciably influence the behavior, either static or dynamic, of the liquid bridge $[2,12,13]$.

In addition, great care was taken to avoid large temperature change rates during experiments. The difference between initial and end-of-run temperatures during the different experiments performed ranged from 0.2 to $6 \mathrm{~K}$ (this difference being smalier as the liquid bridge slenderness increases) and the time spent on each experiment was the appropriate time to keep the temperature change rates between $10^{-3}$ and $10^{-4} \mathrm{~K} \mathrm{~s}^{-1}$. Even more, to avoid large temperature gradients across the tank, in those experiments in which large differences between initial and end-of-run temperatures were expected, the room temperature was also changed according to the surrounding liquid temperature.

\section{Experimental technique and results}

In all experiments the liquid bridge configuration was similar to that represented in fig. 1 (large disk at the top). At the beginning of all experiments the surrounding liquid had methanol in excess, its density being slightly smaller than the working liquid density, aiming to provide a liquid bridge interface with the neck closer to the large disk. The experimental procedure has already been explained in section 1: once a liquid bridge of the desired slenderness and volume is formed, the tank temperature is increased so that the difference $\Delta \rho$ between working liquid density and surrounding liquid density varies. As the Bond number decreases, the liquid-column neck moves towards the bottom disk, the rate of change being small enough to avoid dynamic effects. From time to time photographs of the interface shapes are taken, measuring neck position and Bond number from these pictures later.

To determine the experimental interface shapes, the grid placed at the rear face of the tank provided an accurate reference frame (fig. 4). The pictures were enlarged and the diameter of the liquid column at each horizontal grid line as well as the distance between two reference vertical grid lines were measured. Then, taking into account both scale and conicity effects, the real diameters were calculated. Finally, from these experimental interface shapes, the value of the Bond number was determined by a least square fitting to a second order analytical expression for the interface shape (see appendix). Some experimental liquid bridge interfaces, as measured from the photographs, are compared with analytical ones in fig. 5.

Concerning the experimental results presented here, it must be stated that liquid bridge volumes were measured with a precision of $\pm 0.3 \mathrm{~cm}^{3}$, and the distance between disks with $\pm 0.5 \mathrm{~mm}$. The disk diameters were $30 \mathrm{~mm}$ for the upper disk and $26.9 \mathrm{~mm}(K=0.897)$ or $24 \mathrm{~mm}(K=0.8)$ for the lower disk, the error in disk diameter being \pm 0.1 $\mathrm{mm}$. Therefore, the mean errors in dimensionless variables are $V \pm 0.1$ and $\Lambda \pm 0.02$. In addition, a conservative estimation of the mean error in Bond number and neck position (which was made dimensionless with $L / 2$ ) could be $B \pm 0.0005$ and $z_{\mathrm{n}} \pm 0.03$, respectively.

Experimental results, neck position versus Bond number, are listed in tables 1 and 2 . These results have also been represented in figs. 6 and 7. As it can be observed, liquid bridges behave according to theoretical predictions: as $B$ varies, the neck moves along the liquid column, the neck being at 


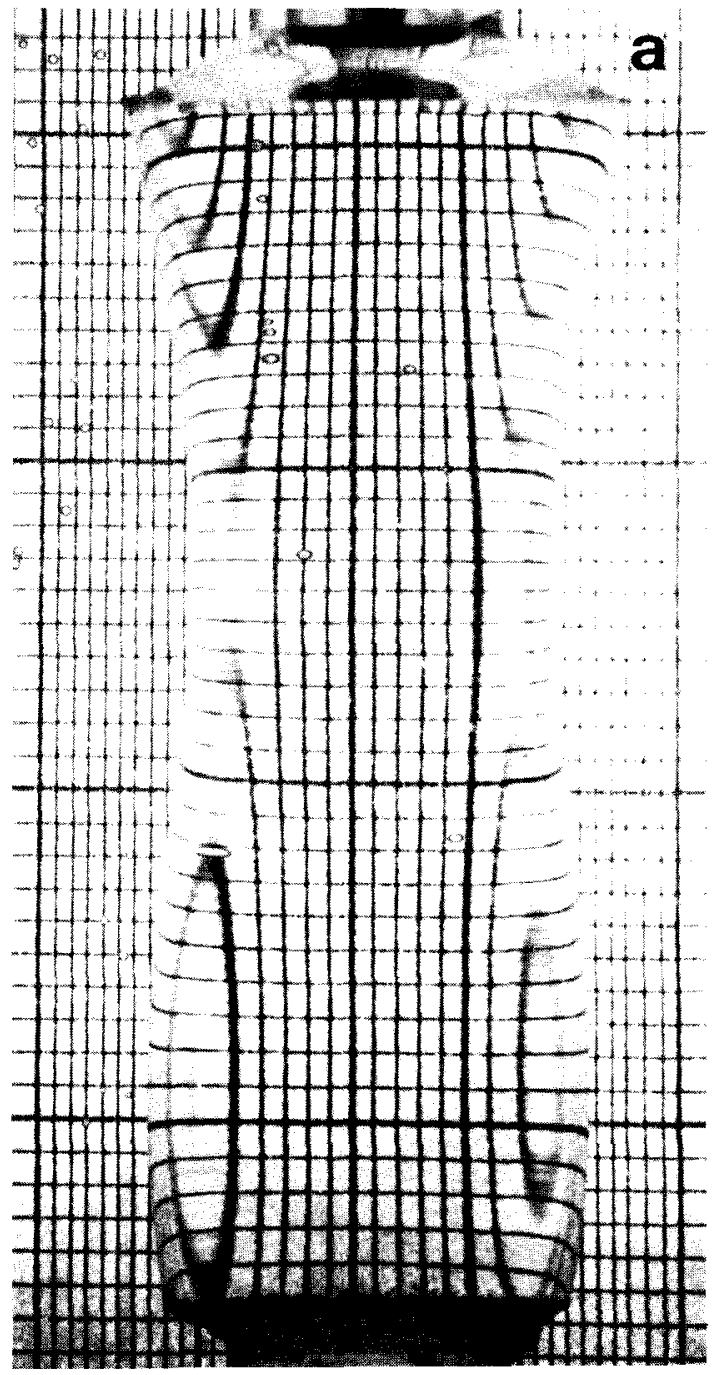

the middle plane for $B=B_{\mathrm{c}}$. Furthermore, $B_{\mathrm{c}}$ results are almost the same no matter which the value of the liquid bridge volume is. The values of $B_{\mathrm{c}}$, as resulting from figs. 6 and 7 , are shown in table 3 . In fig. 8 experimental results, $B_{\text {c }}$ versus $\Lambda$, are compared with the numerical ones calculated in ref. [6], the agreement being reasonably good.

Additional conclusions can be obtained by plotting the dependence on $A$ of $H / B_{\mathrm{c}}$ instead of $B_{\mathrm{c}}$, as shown in fig. 9. As it can be observed, both experimental and numerical results reduce to one single curve when $H / B_{c}$ is used, at least for the

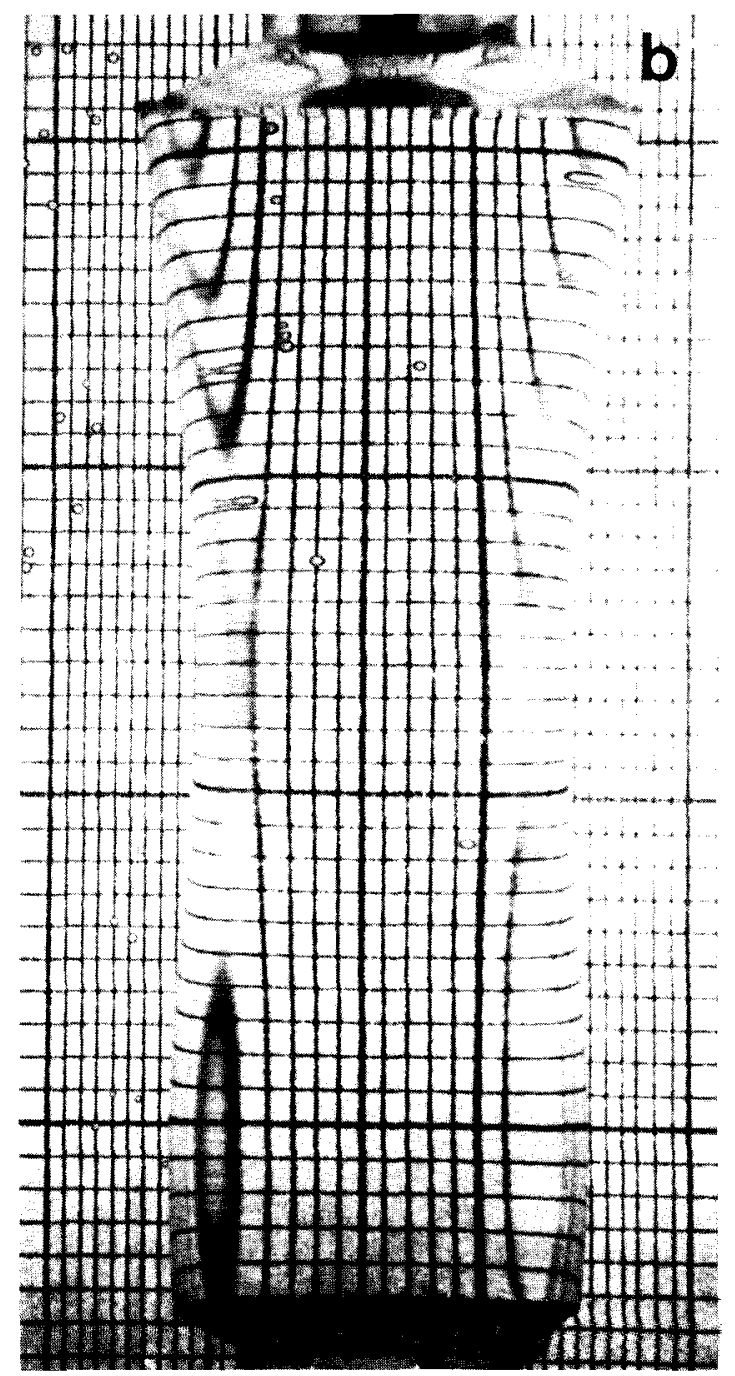

considered values of $H$. In this plot also eq. (1) has been represented. Experimental results lie between numerical and theoretical curves, for each value of $\Lambda$ the experimental value of $H / B_{\mathrm{c}}$ being slightly higher than that numerically calculated. The reason for this difference could be the method used to calculate experimental Bond numbers. In effect, we determine the values of $B$ by fitting experimental interface shapes to a second order analytical expression. The same fittings have been performed by considering only linear terms in the analytical interface shape (these results are not shown here) 

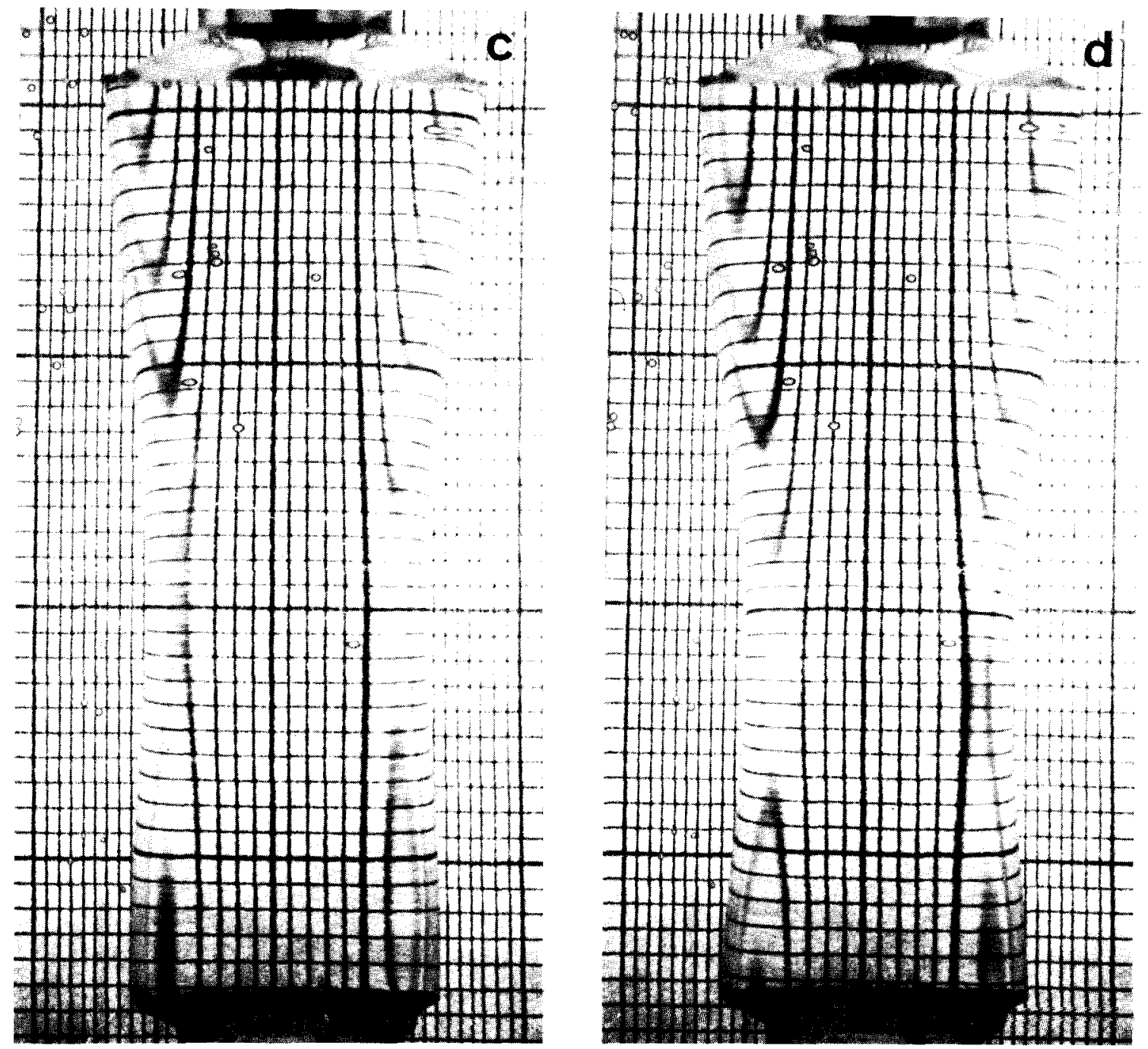

Fig. 4. Photographs of liquid bridges showing the magnifying-glass effect of the liquid column. The photographs correspond to the same liquid bridge configurations shown in fig. 5: $\Lambda=2.63 . K=0.8, V=13.7$ and $B=0.067$ (a), 0.056 (b), 0.048 (c) and 0.039 (d).

and, except in a few cases, it generally results that $B_{\mathrm{s}}>B_{\ell}$, where the subscripts $\mathrm{s}$ and $\ell$ denote second order or linear fitting, respectively. Thus, $H / B_{\mathrm{s}}<H / B_{\ell}$, which seems to indicate that the experimental curve should be even closer to the numerical one if a higher order approximation for the interface shape instead of a second order expression would have been used to determine the value of the experimental Bond numbers.

In this paper only two values of the disk radius ratio ( $K=0.897$ and $K=0.8$ ) have been considered. Although the extension of the experimental study to lower values of $K$ does not mean any practical difficulty (except for the large time involved in neutral buoyancy experimentation), there are two reasons for which those additional experiments were not performed. The first reason concerns the availability of theoretical results $[5,6]$ and the second is related to the experimental data analysis procedure, as the suitability of a second order analytical expression for the interface shape to calculate Bond numbers diminishes when $K$ is quite different from the reference value $K=1$. 

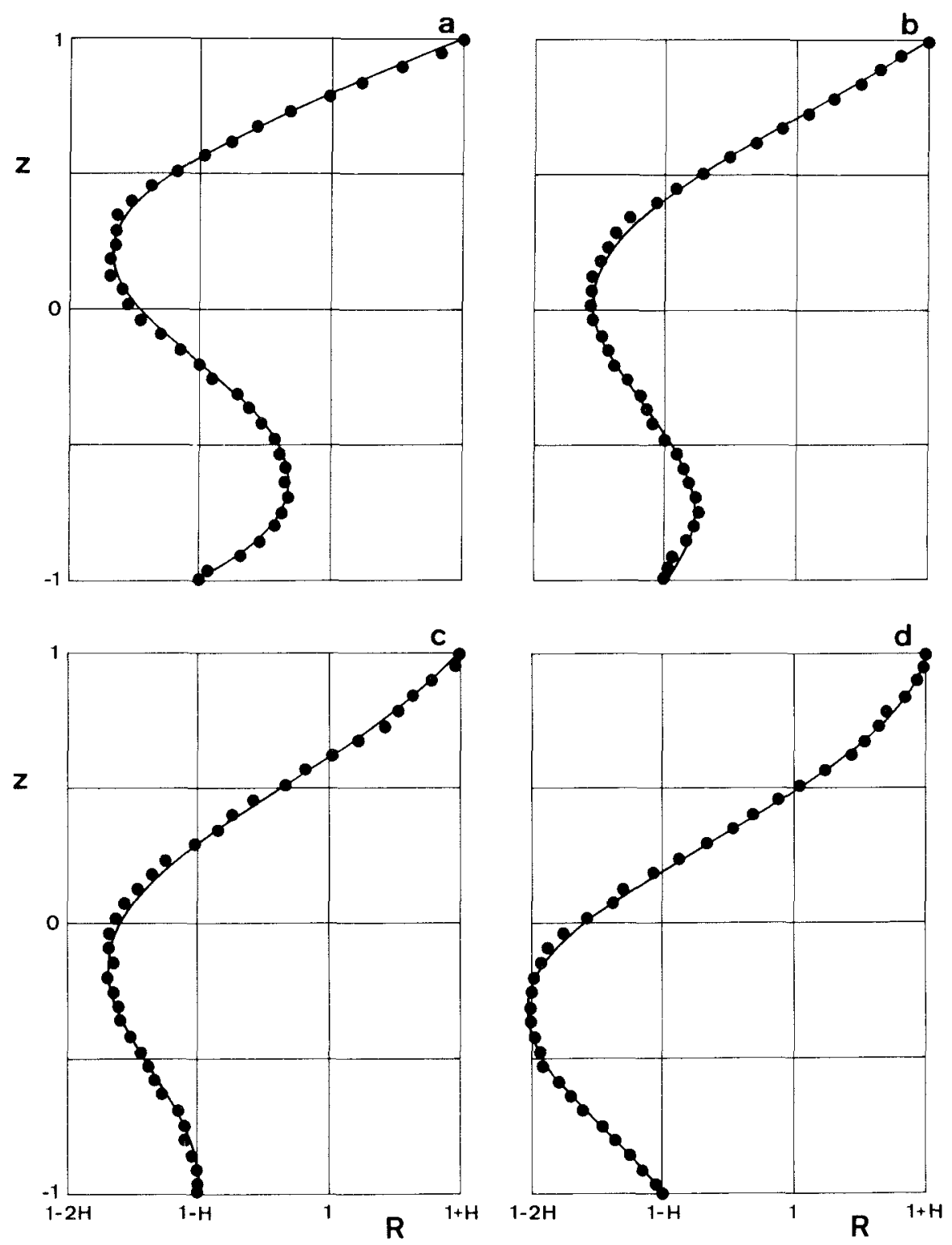

Fig. 5. Liquid bridge interface shapes. In this plot experimental interface shapes (black circles), as measured from photographs shown in fig. 4, are compared with analytical interface shapes (solid lines), as given by eq. (A.2). The results correspond to liquid bridges with $\Lambda=2.63, K=0.8 . V=13.7$ and $B=0.067$ (a), 0.056 (b), 0.048 (c) and 0.039 (d). Note that in each sketch the radial coordinates are considerably enlarged $(H=1 / 9)$. 
Table 1

Variation of the neck position $z_{\mathrm{n}}$ with the Bond number $B$ for liquid bridges with slenderness $A$ and volume $V$, held between unequal disks with a disk radius ratio $K=0.897$

\begin{tabular}{|c|c|c|c|c|c|c|c|c|c|}
\hline \multicolumn{6}{|c|}{$\Lambda=2.79$} & \multicolumn{4}{|l|}{$A=2.60$} \\
\hline \multicolumn{2}{|c|}{$V=17.1$} & \multicolumn{2}{|c|}{$V=16.3$} & \multicolumn{2}{|l|}{$V=15.1$} & \multicolumn{2}{|l|}{$V=16.6$} & \multicolumn{2}{|l|}{$V=14.1$} \\
\hline$B$ & $z_{n}$ & $B$ & $z_{n}$ & $B$ & $z_{11}$ & $B$ & $z_{n}$ & $B$ & $z_{n}$ \\
\hline 0.037 & 0.41 & 0.025 & 0.13 & 0.029 & 0.18 & 0.037 & 0.33 & 0.037 & 0.20 \\
\hline 0.029 & 0.36 & 0.024 & 0.07 & 0.025 & 0.07 & 0.028 & 0.20 & 0.030 & 0.07 \\
\hline 0.027 & 0.31 & 0.023 & -0.05 & 0.024 & 0.03 & 0.027 & 0.09 & 0.027 & 0.00 \\
\hline 0.025 & 0.24 & 0.017 & -0.40 & 0.023 & -0.01 & 0.020 & None & 0.024 & -0.08 \\
\hline 0.023 & 0.04 & & 0.022 & -0.07 & 0.015 & None & 0.016 & -0.22 & \\
\hline 0.022 & None & & 0.020 & -0.12 & -0.007 & -0.57 & & & \\
\hline \multicolumn{6}{|c|}{$\Lambda=2.21$} & \multicolumn{4}{|l|}{$\Lambda=1.79$} \\
\hline \multicolumn{2}{|c|}{$V=13.7$} & \multicolumn{2}{|c|}{$V=12.1$} & \multicolumn{2}{|l|}{$V=10.6$} & \multicolumn{2}{|l|}{$V=11.0$} & \multicolumn{2}{|l|}{$V=8.8$} \\
\hline $\bar{B}$ & $\overline{z_{\mathrm{n}}}$ & $B$ & $z_{\mathrm{n}}$ & $\bar{B}$ & $z_{\mathrm{n}}$ & $B$ & $z_{\mathrm{n}}$ & $B$ & $z_{n}$ \\
\hline 0.162 & 0.49 & 0.084 & 0.30 & 0.068 & 0.13 & 0.240 & 0.47 & 0.221 & 0.28 \\
\hline 0.071 & 0.35 & 0.058 & 0.13 & 0.058 & 0.09 & 0.164 & 0.39 & 0.124 & 0.12 \\
\hline 0.047 & 0.20 & 0.036 & -0.09 & 0.044 & 0.01 & 0.081 & 0.05 & 0.079 & 0.00 \\
\hline 0.035 & None & 0.026 & -0.17 & 0.023 & -0.13 & 0.048 & None & 0.046 & -0.13 \\
\hline 0.006 & -0.66 & 0.016 & -0.25 & -0.031 & -0.35 & -0.020 & -0.66 & -0.050 & -0.30 \\
\hline
\end{tabular}
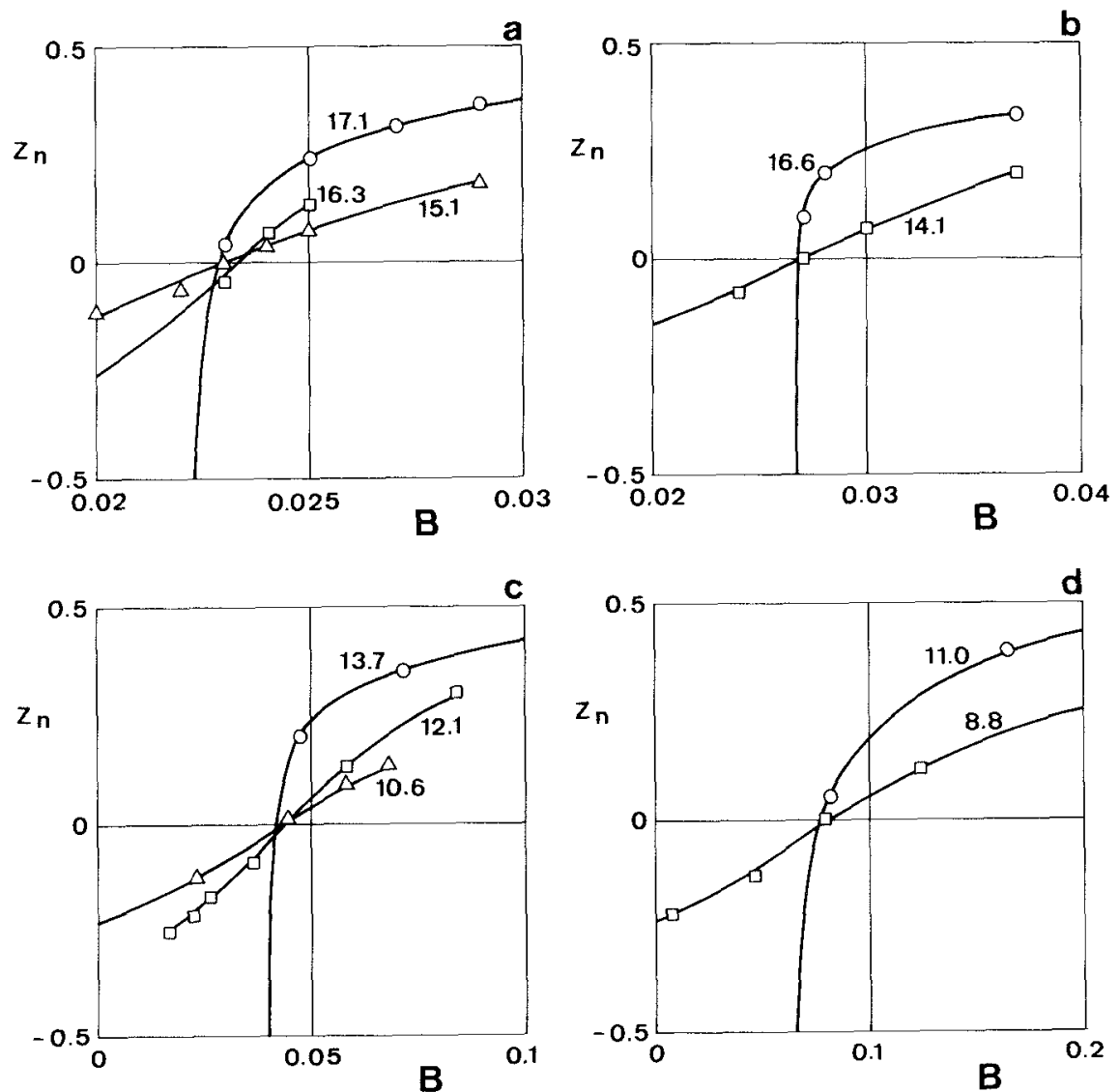

Fig. 6. Experimental results, neck position $z_{\mathrm{n}}$ versus Bond number $B$. The results correspond to liquid bridges with $K=0.897$ and $\Lambda=2.79$ (a). 2.60 (b), 2.21 (c) and 1.79 (d). The numbers on the curves indicate the liquid bridge volume. 
Table 2

Variation of the neck position $z_{n}$ with the Bond number $B$ for liquid bridges with slenderness $A$ and volume $V$, held between unequal disks with a disk radius ratio $K=0.800$

\begin{tabular}{|c|c|c|c|c|c|c|c|c|c|}
\hline \multicolumn{4}{|c|}{$A=2.83$} & \multicolumn{6}{|c|}{$\Lambda=2.63$} \\
\hline \multicolumn{2}{|c|}{$V=15.9$} & \multicolumn{2}{|c|}{$V=14.8$} & \multicolumn{2}{|c|}{$V=16.4$} & \multicolumn{2}{|l|}{$V=14.8$} & \multicolumn{2}{|c|}{$V=13.7$} \\
\hline$B$ & $z_{\mathrm{n}}$ & $B$ & $z_{n}$ & $B$ & $z_{n}$ & $B$ & $z_{n}$ & $B$ & $z_{n}$ \\
\hline 0.057 & 0.33 & 0.051 & 0.17 & 0.069 & 0.39 & 0.072 & 0.28 & 0.067 & 0.18 \\
\hline 0.048 & 0.11 & 0.049 & 0.10 & 0.060 & 0.28 & 0.055 & -0.02 & 0.060 & 0.06 \\
\hline 0.046 & 0.01 & 0.047 & 0.04 & 0.055 & 0.08 & 0.046 & -0.20 & 0.056 & 0.01 \\
\hline 0.044 & -0.12 & 0.046 & -0.02 & 0.050 & None & 0.037 & -0.39 & 0.048 & -0.14 \\
\hline 0.042 & -0.20 & 0.044 & -0.10 & 0.043 & None & & & 0.039 & -0.33 \\
\hline 0.038 & -0.35 & 0.040 & -0.28 & & & & & & \\
\hline \multicolumn{4}{|c|}{$\Lambda=2.44$} & \multicolumn{6}{|c|}{$\Lambda=1.82$} \\
\hline \multicolumn{2}{|c|}{$V=13.0$} & \multicolumn{2}{|c|}{$V=12.0$} & \multicolumn{2}{|c|}{$V=10.2$} & \multicolumn{2}{|l|}{$V=9.6$} & \multicolumn{2}{|c|}{$V=8.2$} \\
\hline$B$ & $z_{\mathrm{n}}$ & $B$ & $z_{\mathrm{n}}$ & $B$ & $z_{n}$ & $B$ & $z_{n}$ & $B$ & $z_{\mathrm{n}}$ \\
\hline 0.086 & 0.16 & 0.067 & 0.00 & 0.227 & 0.33 & 0.172 & 0.12 & 0.201 & 0.12 \\
\hline 0.069 & 0.06 & 0.063 & -0.04 & 0.178 & 0.20 & 0.086 & -0.20 & 0.182 & 0.08 \\
\hline 0.066 & 0.00 & 0.056 & -0.10 & 0.126 & -0.20 & 0.010 & -0.43 & 0.156 & 0.03 \\
\hline 0.063 & -0.07 & 0.044 & -0.21 & 0.105 & -0.36 & -0.048 & -0.49 & 0.109 & -0.06 \\
\hline 0.056 & -0.22 & & & & & & & 0.046 & -0.23 \\
\hline 0.049 & -0.30 & & & & & & & & \\
\hline
\end{tabular}
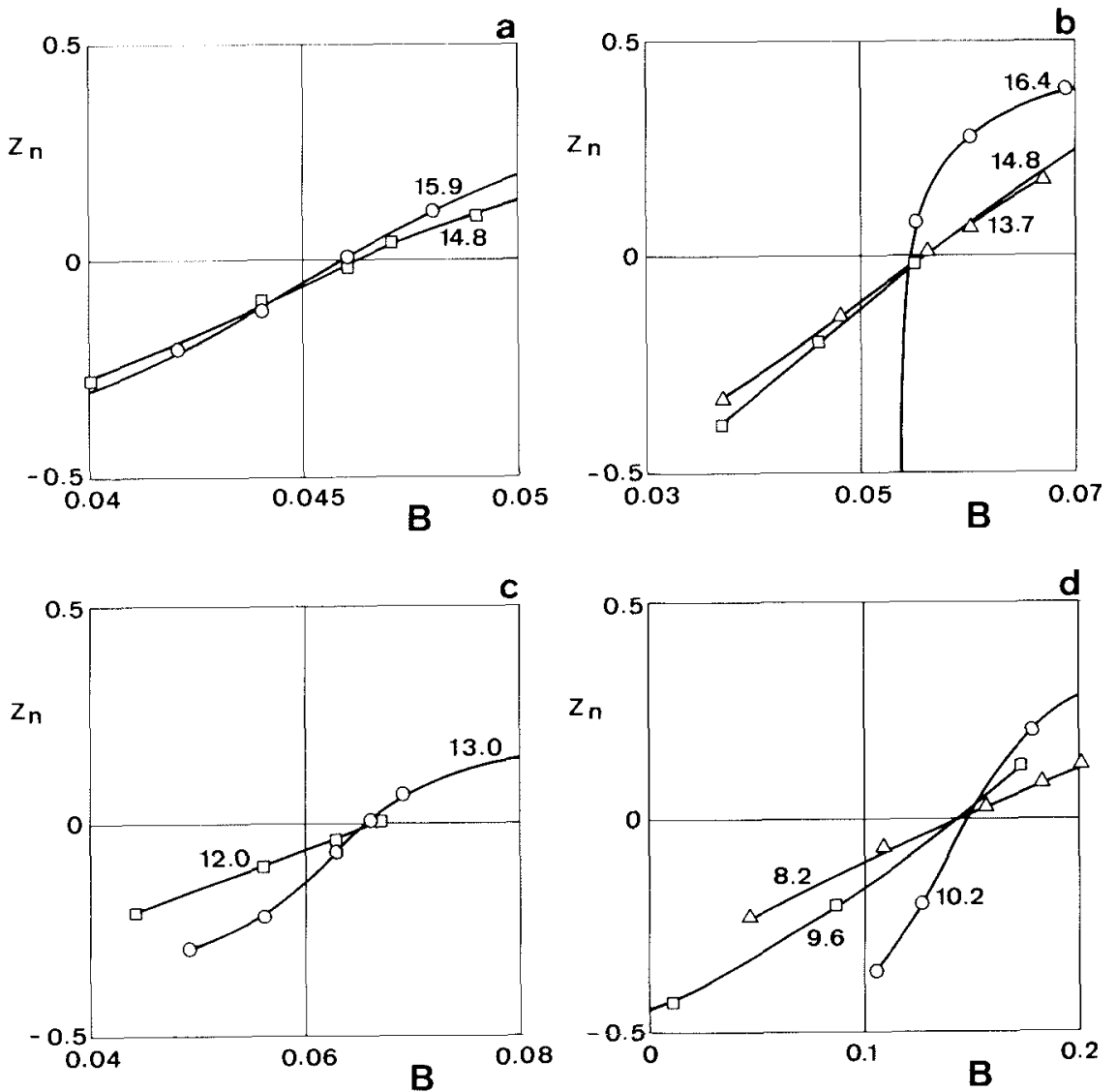

Fig. 7. Experimental results, neck position $z_{\mathrm{n}}$ versus Bond number $B$. The results correspond to liquid bridges with $K=0.8$ and $A=2.83$ (a), 2.63 (b), 2.44 (c) and 1.82 (d). The numbers on the curves indicate the liquid bridge volume. 


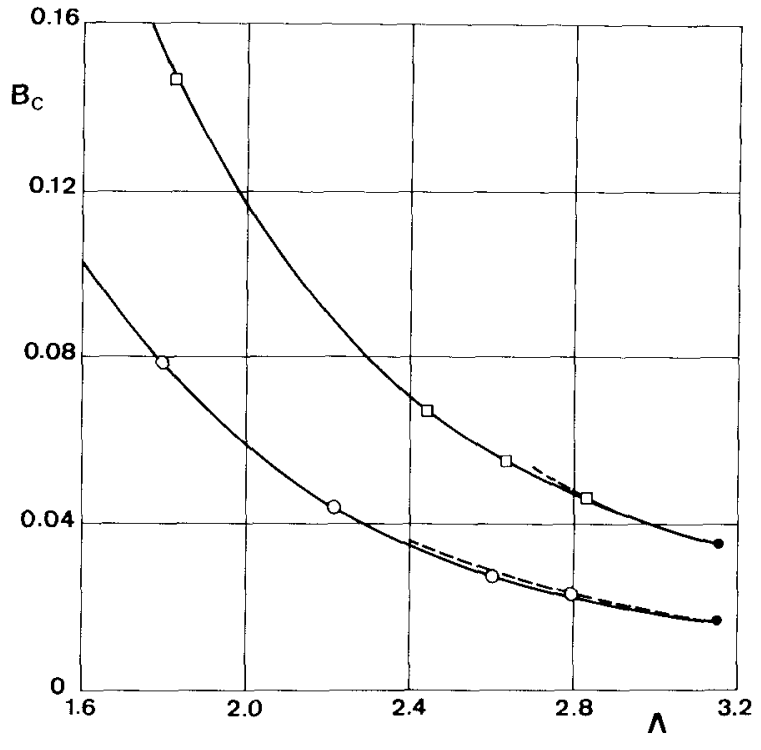

Fig. 8. Variation with the slenderness $A$ of the Bond number $B_{c}$ at which an absolute minimum volume stability limit is reached. The solid lines correspond to experimental results and the dashed lines to numerical results calculated in [6]. The symbols indicate the value of the disk radius ratio, $K=0.897$ ( $O)$ and $K=0.8(\square)$.

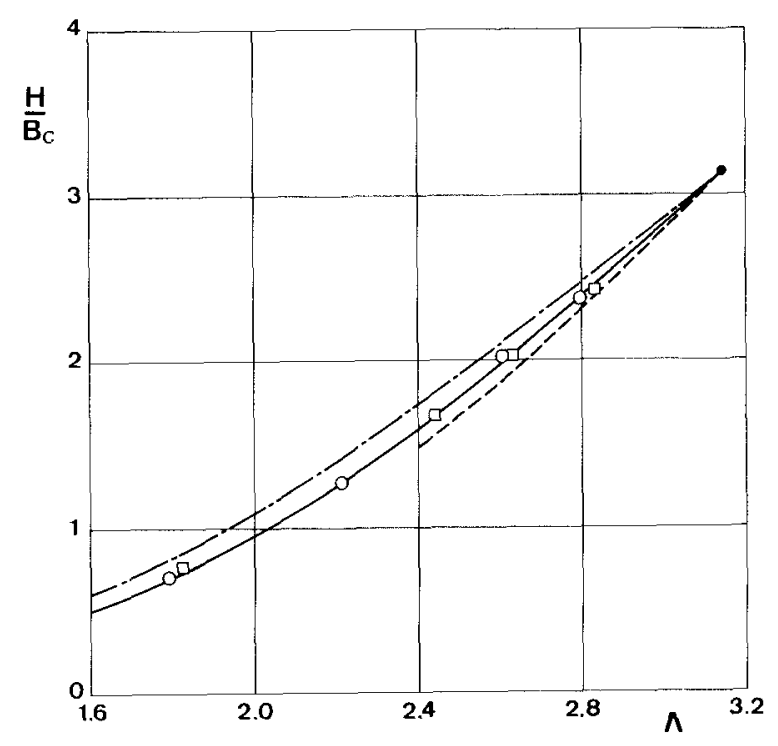

Fig. 9. Variation with the slenderness $A$ of the ratio $H / B_{\text {c. }}$. The solid line corresponds to experimental results (the symbols indicate the value of the disk radius ratio, $K=0.897(0)$ and $K=0.8(\square)$ ), whereas the dashed line corresponds to numerical results calculated in ref. [6] and the dot-dashed line to eq. (1).
Table 3

Variation with the slenderness $A$ of the Bond number $B_{6}$ at which a minimum volume stability limit is reached, and the ratio $H / B_{c}$, where $H=(1-K) /(1+K), K$ being the disk radius ratio

\begin{tabular}{|c|c|c|c|c|c|}
\hline \multicolumn{3}{|c|}{$K=0.897$} & \multicolumn{3}{|c|}{$K=0.800$} \\
\hline$\Lambda$ & $B_{\mathrm{c}}$ & $H / B_{\mathrm{c}}$ & $\bar{\Lambda}$ & $B_{i}$ & $H / B_{C}$ \\
\hline 2.79 & 0.023 & 2.37 & 2.83 & 0.046 & 2.42 \\
\hline 2.60 & 0.027 & 2.02 & 2.63 & 0.055 & 2.02 \\
\hline 2.21 & 0.043 & 1.27 & 2.44 & 0.067 & 1.66 \\
\hline 1.79 & 0.078 & 0.70 & 1.82 & 0.146 & 0.76 \\
\hline
\end{tabular}

\section{Acknowledgement}

This paper has been supported by the Spanish National Commission for Space Research (CONIE).

\section{Appendix}

In this part a second order analytical expression for the liquid bridge equilibrium interface shape is calculated. The formulation of the problem has already been presented in section 2, eqs. (2) to (4). To calculate equilibrium interface shapes the following small parameters are defined

$\epsilon_{1}=\frac{V}{2 \pi \Lambda}-1$,

$\epsilon_{2}=B$,

$\epsilon_{3}=2 H$.

The first parameter measures the deviation of the liquid bridge volume from cylindrical volume, the second is the Bond number, and the third is related to the boundary conditions: $S( \pm \Lambda)=1 \pm \epsilon_{3}$ $+\frac{1}{4} \epsilon_{3}^{2}$, which is similar to eq. (3).

Let us to introduce the following expansion for the variable $S(z)$

$S(z)=1+\sum_{i} \epsilon_{i} s_{i}(z)+\sum_{i, j} \epsilon_{i} \epsilon_{j} s_{i j}(z)$,

with $s_{i j}(z)=s_{j i}(z)$. Then, introducing eq. (A.2) in (2)-(4) and equating equal power terms of $\epsilon_{i}$ yields the following set of problems. 
$\epsilon_{1}$ order:

$s_{1}+s_{1: z}+p_{1}=0$,

$s_{1}( \pm \Lambda)=0$,

$\int_{-\Lambda}^{\Lambda} s_{1} \mathrm{~d} z=2 \Lambda$

$\epsilon_{2}$ order:

$s_{2}+s_{2, .}-2 z+p_{2}=0$.

$s_{2}( \pm \Lambda)=0$,

$\int_{-1}^{\Lambda} s_{2} \mathrm{~d} z=0$;

$\epsilon_{3}$ order:

$s_{3}+s_{3: 3}+p_{3}=0$,

$s_{3}( \pm \Lambda)= \pm 1$,

$\int_{-A}^{A} s_{3} \mathrm{~d} z=0$

With these three linear problems, volume and microgravity requirements are met, but not boundary conditions at the disks. Therefore, all second order problems will have homogeneous boundary conditions, except that concerning $s_{33}(z)$.

$\epsilon_{i} \epsilon_{\text {, }}$ order:

$s_{i j}+s_{i j_{z=}}=\frac{1}{4}\left(s_{i_{z}} s_{j_{z}}+s_{i} s_{j z z}+s_{i z_{z z}} s_{j}+3 s_{i} s_{j}\right)-p_{i j}$,

$s_{i j}( \pm \Lambda)=\frac{1}{4} \delta_{i 3} \delta_{j 3}$,

$\int_{-\Lambda}^{\Lambda} s_{i j} \mathrm{~d} z=0$

where $\delta_{i j}$ stands for the Kronecker delta function. The solutions of the linear problems (already calculated in ref. [7]) are

$s_{1}=N(\cos z-\cos \Lambda)$,

$s_{2}=2(z-\Lambda \sin z / \sin \Lambda)$,

$s_{3}=\sin z / \sin \Lambda$;

with $N=\Lambda /(\sin \Lambda-\Lambda \cos \Lambda)$. Then, after substituting these expressions in the second order problems, the following solutions are obtained

$s_{11}=T_{11}(z)-T_{11}(\Lambda)$,
$s_{12}=T_{12}(z)-T_{12}(\Lambda) \sin z / \sin \Lambda$,

$s_{13}=T_{13}(z)-T_{13}(\Lambda) \sin z / \sin \Lambda$,

$s_{22}=T_{22}(z)-T_{22}(\Lambda)$,

$s_{23}=T_{23}(z)-T_{23}(\Lambda)$,

$s_{33}=\frac{1}{4}\left(1-s_{1}\right)$,

where the functions $T_{i j}$ are

$T_{11}(z)=\frac{1}{2} N^{2} \cos \Lambda[(1-N \Lambda \sin \Lambda) \cos z$ $-z \sin z]$,

$T_{12}(z)=\frac{1}{2} N\left[\frac{1}{2} z^{2} \sin z+\frac{\Lambda}{N \sin \Lambda} z \cos z\right.$

$-3 z \cos \Lambda]$

$T_{13}(z)=\frac{N \cos \Lambda}{4 \sin \Lambda} z \cos z$,

$T_{22}(z)=\frac{\Lambda}{\sin \Lambda}\left[\left(4-\Lambda^{2}+z^{2}\right) \cos z-2 z \sin z\right]$

$+3 z^{2}$

$T_{23}(z)=\frac{-1}{4 \sin \Lambda}\left[\left(4-\Lambda^{2}+z^{2}-2 N \Lambda \sin \Lambda\right)\right.$

$\times \cos z-2 z \sin z]$.

\section{References}

[1] I. Da Riva and L.G. Napolitano, in: Material Sciences under Microgravity, ESA SP-191 (ESA, Paris, 1983) p. 5.

[2] J. Meseguer and A. Sanz, J. Fluid Mech. 153 (1985) 83.

[3] L.H. Ungar and R.A. Brown, Phil. Trans. Roy. Soc. London A306 (1982) 347.

[4] I. Martínez, in: Material Sciences under Microgravity, ESA SP-191 (ESA, Paris, 1983) p. 267.

[5] J. Meseguer, J. Crystal Growth 67 (1984) 141.

[6] J. Meseguer, J. Crystal Growth, to be published.

[7] J. Meseguer, in: Material Sciences under Microgravity, Results of Spacelab-1, ESA SP-222 (ESA, Paris, 1984) p. 297.

[8] G.C. Mason, J. Colloid Interface Sci. 32 (1970) 172.

[9] J.R. Carruthers and M. Grasso, J. Crystal Growth 13/14 (1972) 611.

[10] S.R. Coriell, S.C. Hardy and M.R. Cordes, J. Colloid Interface Sci. 60 (1977) 126.

[11] M.P. Elagin, A.P. Lebedev and A.V. Tsmelev, in: Hydromechanics and Heat and Mass Transfer (Nauka, Moscow, 1982) p. 24 (in Russian).

[12] A. Sanz and I. Martinez, J. Colloid Interface Sci. 93 (1983) 235 . 
[13] A. Sanz, J. Fluid Mech. 156 (1985) 101.

[14] J.M. Haynes, Bull. Mater. Sci. 4 (1982) 75.

[15] D. Rivas and J. Meseguer, J. Fluid Mech. 138 (1984) 417.

[16] 1. Martinez and D. Rivas, Acta Astron. 9 (1982) 339.
[17] R. Tagg, L. Cammack, A. Croonquist and T.G. Wang, in: Rotating Liquid Drops: Plateau's Experiment Revisited, JPL 900-954, Pasadena, CA, 1980. 